\title{
sciendo
}

\section{Effects of Resisted vs. Conventional Sprint Training on Physical Fitness in Young Elite Tennis Players}

\author{
by \\ Manuel Moya-Ramon', Fabio Yuzo Nakamura², Anderson Santiago Teixeira ${ }^{3}$, \\ Urs Granacher ${ }^{4}$, Francisco Javier Santos-Rosa ${ }^{5}$, David Sanz-Rivas ${ }^{6}$, \\ Jaime Fernandez-Fernandez $z^{6,7}$
}

This study aimed to compare the effects of 6-week resisted sprint (RST) versus conventional (unresisted) sprint training (CG) on sprint time, change of direction (COD) speed, repeated sprint ability (RSA) and jump performance (countermovement jump (CMJ) and standing long jump (SLJ)) in male young tennis players. Twenty players (age: $16.5 \pm 0.3$ years; body mass: $72.2 \pm 5.5 \mathrm{~kg}$; body height: $180.6 \pm 4.6 \mathrm{~cm})$ were randomly assigned to one of the two groups: RST $(n=10)$ and CG $(n=10)$. The training program was similar for both groups consisting of acceleration and deceleration exercises at short distances (3-4 $\mathrm{m}$ ), and speed and agility drills. The RST group used weighted vests or elastic cords during the exercises. After 6 weeks of intervention, both training regimes resulted in small-to-moderate improvements in acceleration and sprint ability $(5,10,20 \mathrm{~m})$, SLJ and CMJ performances, COD pivoting on both, the non-dominant (moderate effect) and the dominant (small effect) foot, and the percentage of decrement (small effects) during a RSA test. Between-group comparisons showed that the SLJ $(\Delta=2.0 \%)$ and $5 \mathrm{~m}$ sprint time $(\Delta=1.1 \%)$ improved more in the RST group compared with the CG group. This study showed that 6 weeks of RST or unresisted training are time-efficient training regimes for physical improvements in young male tennis players.

Key words: young athletes, sprint performance, tennis, power.

\section{Introduction}

Tennis match play is characterized by intermittent whole body efforts with short (2-10 s) bouts of high-intensity exercise during rallies followed by short (10-20 s) recovery bouts between rallies and a longer rest period between games (60-90 s). Overall, this results in an average match time of $\sim 1.5 \mathrm{~h}$ (Fernandez-Fernandez et al., 2009; Kovacs, 2006, 2007). After serving the ball with a velocity of $180-200 \mathrm{~km} \cdot \mathrm{h}^{-1}$, a tennis player needs to accelerate not only in a straight line, but also laterally and multi-directionally. In other words, rapid stop and go movements together with quick change of directions (CODs) constitute major performance determinants in tennis (Fernandez-Fernandez et al., 2014). Consequently, the development and design of training regimes

\footnotetext{
1 - Department of Sports Sciences, Miguel Hernandez University, Elche, Spain.

2 - Department of Medicine and Aging Sciences, "G. d'Annunzio" University of Chieti-Pescara, Chieti, Italy; The College of Healthcare Sciences, James Cook University, Townsville, Australia; Associate Graduate Program in Physical Education UPE/UFPB, João Pessoa, PB, Brazil.

3 - Physical Effort Laboratory, Sports Center, Federal University of Santa Catarina, Florianópolis - SC, Brazil; Research Group for Development of Football and Futsal, Sports Center, Federal University of Santa Catarina, Florianópolis - SC, Brazil.

4 - Division of Training and Movement Sciences, Research Focus Cognition Sciences, University of Potsdam, Potsdam, Germany.

5 - Faculty of Sport, Pablo de Olavide University, Seville, Spain.

6 - Spanish Tennis Federation, Madrid, Spain.

7 - Department of Physical Activity and Sport Sciences, Universidad de León, Spain.
} 
that have the potential to enhance these sportspecific fitness qualities are of significant interest to tennis coaches as well as strength and conditioning specialists.

There is evidence in the literature that different training protocols are effective in improving jump, sprint and COD performances (Loturco et al., 2017). Moreover, it has been reported that training-induced enhancements in muscle strength and power translate into sprint and COD performances (Cormie et al., 2011). Of note, specific strength exercises have been implemented in sprint training routines. This is also known as resisted sprint training (RST) (Hrysomallis, 2012; Petrakos et al., 2016). Previous research has shown that RST improves maximum strength and sprint performances (Cottle et al., 2014; Martínez-Valencia et al., 2015). However, findings in the literature are controversial. A previous systematic review showed no additional effects of RST on linear sprint speed compared with conventional or unresisted sprint training regimes (Petrakos et al., 2016). The observed discrepancies in previous research are most likely due to the large methodological heterogeneity with regard to the included resistive sprint devices. While some studies used weighted sleds or weighted vests (Carlos-Vivas et al., 2018; Clark et al., 2010), others used parachutes or elastic cables (Gil et al., 2018; Loturco et al., 2017). In addition, ineffective overload principles were implemented in the respective training regimes (Gil et al., 2018). Despite this controversy in the literature, there are few studies with tennis players that illustrate the positive effects of conventional sprint (i.e., repeated sprint) training on physical fitness (i.e., linear sprint speed, jump and COD performances) in junior elite (Fernandez-Fernandez et al., 2015) or moderately trained tennis players (Fernandez-Fernandez et al., 2012). However, to the best of our knowledge, no previous study has analyzed the effects of RST compared with unresisted sprint training on physical fitness in young tennis players.

Therefore, the aim of this study was to contrast the effects of RST versus conventional (i.e., unresisted) linear sprint training on linear sprint and COD performances as well as lowerlimb power (i.e., countermovement jump and standing long jump) in young tennis players. Based on the study of Gil et al. (2018), we hypothesized that RST would be more effective in improving specific physical fitness compared with the unresisted sprint training in tennis players.

\section{Methods}

\section{Participants}

Twenty competitive male junior tennis players (mean \pm SD; age: $16.5 \pm 0.3$ years; body mass: $72.2 \pm 5.5 \mathrm{~kg}$; body height: $180.6 \pm 4.6 \mathrm{~cm}$ ) with an international ranking between 150 and 300 (International Tennis Federation ranking) participated in this study. Players were divided into a resisted training group (RST; $\mathrm{n}=10$; mean \pm SD; age: $16.7 \pm 0.1$ years; body mass: $72.0 \pm 5.2 \mathrm{~kg}$; body height: $181.6 \pm 4.8 \mathrm{~cm}$ ) and a conventional group (CG; $\mathrm{n}=10$; mean $\pm \mathrm{SD}$; age: $16.4 \pm 0.3$ years; body mass: $71.1 \pm 7.2 \mathrm{~kg}$; body height: 179.9 $\pm 4.4 \mathrm{~cm})$. The mean training background of the players was $9.0 \pm 2.6$ years, which focused on tennis-specific training (i.e., technical and tactical skills), aerobic and anaerobic training (i.e., onand off-court exercises), and basic strength training. They were all free of cardiovascular and pulmonary diseases and were not taking any medication. Written informed consent was obtained from players and their parents/legal representatives. The study was approved by the institutional Research Ethics Committee (Coaches Education and Research Area, Spanish Tennis Federation (RFET); reference: RFET2019-RS1), and conformed to the recommendations of the latest version of the Declaration of Helsinki.

\section{Measures}

Sprint Test

Running speed was evaluated using the 20-m linear sprint test from a standing start with 5 and $10 \mathrm{~m}$ split times (Time It; Eleiko Sport, Halmstad, Sweden). Each sprint was initiated from an individually chosen standing position, 50 $\mathrm{cm}$ behind the photocell gate, which started a digital timer. Each player performed 3 maximal 20-m sprints, separated by at least $2 \mathrm{~min}$ of passive recovery. The best performance was recorded for further analysis. The intraclass correlation coefficient (ICC) for the time of this sprint test was 0.87 .

Modified 5-0-5 Agility Test (COD test)

The abilities of athletes to perform a single, rapid $180^{\circ}$ change of direction over a $5 \mathrm{~m}$ distance was measured using a modified version 
(stationary start) of the 5-0-5 agility test (GalloSalazar et al., 2017). Players started in a standing position with their preferred foot behind the starting line. Thereafter they accelerated in a forward direction without a racket at maximal effort. One trial pivoting on both the dominant (505 DOM) and the non-dominant limb (505 ND) was completed and the best time recorded to the nearest $0.01 \mathrm{~s}$ (Time It; Eleiko Sport, Halmstad, Sweden). Two minutes of rest were allowed between trials. The ICC of this test was 0.92 .

Vertical Jump Test

The vertical jump is a common action in many sports. Biomechanically it is similar to game-related dynamic and vertical movements (Girard et al., 2005; Markovic et al., 2004). Thus, it is important to include some type of vertical-jump assessment to evaluate explosive power in tennis. Accordingly, a countermovement jump (CMJ) without an arm swing was performed on a contact platform (Ergojump ${ }^{\circledR}$, Finland). Each player performed 3 maximal CMJs interspersed with $45 \mathrm{~s}$ of passive recovery, and the best trial with highest jump height was used for further analysis (Markovic et al., 2004). The ICC of the jump height for this test was 0.92 .

Standing Long Jump (SLJ) Test

For the SLJ, players stood behind a starting line with feet shoulder width and placed together. They pushed off vigorously and jumped forward for maximal distance. The distance was measured from the take-off line to the point where the back of the heel nearest to the take-off line landed on the ground. The best (in $\mathrm{cm}$ ) out of 2 trials was used for subsequent statistical analysis (Castro-Piñero et al., 2010). The ICC of the jump distance for this test was 0.78 .

Repeated-Sprint Ability (RSA) Shuttle Test

To measure RSA, we used a test consisting of ten $21-\mathrm{m}$ shuttle sprints (i.e., $5 \mathrm{~m}+11$ $\mathrm{m}+5 \mathrm{~m}$ ), which was designed to measure both repeated sprint and COD abilities. The test was conducted in accordance with a previous study (Fernandez-Fernandez et al., 2012). Players stood with their racket in a frontal position in the middle of the baseline focusing the net. Upon an acoustic signal, players turned sideway and ran to the prescribed backhand (left) or the forehand (right) corner. Players were instructed to run forward in a straight line and turn around $\left(180^{\circ}\right)$ as their feet touched the line of the turning point and their racket a cone on the line. After having touched the first cone with the racket, athletes returned to the opposite side of the court by running forward. There they touched the second cone with the racket, turned around and ran to the starting position. After $15 \mathrm{~s}$ of passive recovery, players started again. Each shuttle sprint time was measured using a photocell system (Time It; Eleiko Sport, Halmstad, Sweden). The mean time and the percent decrement score during the RSA test were calculated. Each player completed a preliminary single shuttle sprint test, which was used as a criterion score for the subsequent shuttle sprint test. After the first preliminary single shuttle sprint, players rested for $5 \mathrm{~min}$ before the start of the RSA test. If performance during the first RSA trial was worse than the criterion score (i.e., $2.5 \%$ longer time to complete the test), the test was immediately terminated and athletes were asked to repeat the RSA test at maximum effort after a 5-min rest period (Fernandez-Fernandez et al., 2012; Spencer et al., 2005). ICC values for best RSA time (RSAbest), mean RSA time (RSAmean), and percentage of decrement $(\% \mathrm{Dec})$ were $0.81,0.73$ and 0.49 , respectively.

Design and procedures

The 20 tennis players involved in the study were matched and allocated into a RST and a conventional group (CG) and were tested before and after a 6-week specific conditioning program. The intervention took place at the beginning of the summer competition season (April to May). Single and double tennis matches were played every weekend during the experimental period. After appropriate familiarization (i.e., completion of a full testing session 1 week before pretests), the physical fitness tests were completed 1 week before and after the training period. During the intervention period, both groups, RST and CG, performed 2 training sessions per week in addition to their regular training regimes for 6 consecutive weeks. Sessions were separated by 48 $\mathrm{h}$ to allow sufficient recovery time. RST and CG sprint training was conducted at the beginning of the training session after a short standardized 8 to 10-min dynamic warm-up and prior to the tennisspecific session.

To reduce the interference of uncontrolled variables, all participants were instructed to maintain their habitual lifestyle and normal 
dietary intake before and during the study. Players were told not to exercise on the day before a test and to consume their last (caffeine-free) meal at least 2 hours before the scheduled test time. Physical fitness tests were conducted at the same time of day during pre- and post-tests. Participants had to complete at least $85 \%$ of the training sessions and all tests were included in the final data analyses.

Specifically, the training program consisted of a combination of acceleration and deceleration movements at short distances (3-4 m) and speed/agility drills (8-10 s) without any additional load/resistance (CG) or using weighted vests (WVs) (Kettler, Germany) and elastics cords (ECs) (SKLZ, Durham, USA) (RST). Due to the complexity of supervising the tennis-specific training program, coaches organized weekly meetings to assign similar tennis training loads to both RST and CG groups (i.e., number of exercises, technical/tactical aims). Both groups completed the same training (consisting of forward, backward and multidirectional sprints, with 1 to 6 changes of directions [COD]), interspersed with $25 \mathrm{~s}$ of active recovery between repetitions and 2-3 min rest intervals between sets) (Table 1). The only difference between the two interventions was that the RST group performed the exercises requiring more CODs with a weighted vest that corresponded to $10-15 \%$ of each individual's body mass (a moderate load according to a prior study; Petrakos et al., 2016). The RST group additionally used a medium resistance elastic cord which was fixated around the athletes' waist offering resistance during exercises requiring less CODs. Following a previous study (Gil et al., 2018), the overload in the latter training mode reduced sprinting performance to the nearest of $10 \%$ in comparison to the unresisted condition (Hrysomallis, 2012). As inappropriate overload may alter movement technique, and consequently the magnitude of chronic adaptations, the additional overload was kept constant throughout the experimental period. Both groups, RST and CG, followed their normal tennis training (4-5 $\times$ week), in addition to 2 self-regulated low- to moderate-intensity injury prevention (e.g., core training, shoulder and hip strengthening, and flexibility) sessions.

\section{Statistical Analyses}

Data are presented as means and standard deviations ( \pm SD) or $\pm 90 \%$ confidence intervals $( \pm 90 \%$ CI $)$. First, training-induced adaptations were compared using a two-way repeated measure ANOVA with one between factor (RST vs. CG) and one within factor (pretraining vs. post-training). When a significant $F$ value was detected, Bonferroni post hoc procedures were used. The significance level was set at $p \leq 0.05$. These analyzes were carried out using the SPSS (SPSS 17.0 version, Chicago, Illinois, USA). Second, data were also analyzed for practical significance using magnitude-based inferences (MBI) (Hopkins et al., 2009). To examine the effects of the type of intervention (RST vs. CG) on RSA, change of direction speed, and proxies of lower-limb power, differences between groups (RST vs. CG) and over time (pretraining vs. post-training) were calculated. The smallest worthwhile change (SWC) was calculated $(0.2 \times \mathrm{SD})$ and $90 \%$ CIs were determined. Quantitative chances of beneficial/higher or harmful/lower effects were assessed qualitatively as follows: 25 to $75 \%$, possibly; 75 to $95 \%$, likely; 95 to $99 \%$, very likely; and $>99 \%$, almost certain. If the chance of having beneficial/higher or harmful/lower performances was both $>5 \%$, the true difference was assessed as unclear. In addition, effect sizes (Cohen's d) of changes in physical fitness were calculated (Hopkins et al., 2009). Threshold values for Cohen's d effect size (ES) were $0.20,0.60,1.20,2.0$ and 4.0 for small, moderate, large, very large and extremely large effects, respectively. Pearson correlation coefficients $(r)$ were used to determine the relationships between changes in SLJ and CMJ performances with changes in sprint running performance. The magnitude of relationships was assessed according to the following thresholds: $\leq 0.1$, trivial; >0.1-0.3, small; >0.3-0.5, moderate; $>0.5-0.7$, large; $>0.7-0-9$, very large; and $>0.9-1.0$, almost perfect. Practical inferences of the correlation coefficients were also considered (Hopkins, 2007).

\section{Results}

Table 2 shows the raw data, relative changes, and qualitative outcomes derived from MBI analyses for all physical fitness measurements.

Two-way repeated measures ANOVA

There was no interaction (time vs. 
training group) or main effect for group $(p>0.05)$ for all physical performance variables. After the training intervention, except for RSAbest $(F=3.083$; $p=0.096$ ), a significant main time effect was found for all the other performance outcomes. The following analyzed variables significantly improved from pre- to post-training period in both RST and CG groups: $5 \mathrm{~m}(\mathrm{~F}=33.492$; $p<$ $0.001), 10 \mathrm{~m}(\mathrm{~F}=18.871 ; p<0.001)$ and $20 \mathrm{~m}(\mathrm{~F}=$ 24.308; $p<0.001)$ sprint times, $505 \mathrm{ND}(\mathrm{F}=18.705$; $p<0.001)$ and 505 DOM $(\mathrm{F}=12.627 ; p=0.002)$, SLJ height $(\mathrm{F}=56.091 ; p<0.001)$, CMJ height $(\mathrm{F}=$ 38.764; $p<0.001)$, RSAmean $(\mathrm{F}=10.860 ; p=0.004)$ and $\% \operatorname{Dec}(\mathrm{F}=7.846 ; p=0.012)$.

Magnitude-based inferences approach

Baseline between-group differences were rated as unclear for all performance outcomes. Following training, sprint times improved in both groups and all split times, with decreases in $5 \mathrm{~m}$ (ES $\pm 90 \% \mathrm{CI}$, qualitative descriptor for RST: -0.66 \pm 0.31 , very likely; for CG: $-0.67 \pm 0.28$, very likely), $10 \mathrm{~m}$ (for RST: $-0.32 \pm 0.20$, likely; for CG: $-0.77 \pm$
0.39, very likely), and $20 \mathrm{~m}$ sprint times (for RST: $0.46 \pm 0.33$, likely; for CG: $-0.57 \pm 0.20$, almost certainly).

An enhanced performance was observed after both training regimes for the ability to change directions quickly, with meaningful changes in 505 ND (RST: $-0.60 \pm 0.43$, likely; CG: $0.64 \pm 0.31$, very likely) and 505 DOM (RST: $-0.41 \pm$ 0.28 , likely; CG: $-0.42 \pm 0.29$, likely). Post training, improvements in all measures of jump performance were found for RST and CG groups. Performance enhancements were observed for the SLJ (RST: $0.63 \pm 0.24$, almost certainly; CG: $0.69 \pm$ 0.22 , almost certainly) and the CMJ (RST: $0.39 \pm$ 0.19 , very likely; CG: $0.62 \pm 0.24$, almost certainly). Training-induced changes for RSA performance were detected for \%Dec (RST: $0.43 \pm 0.39$, likely; CG: $0.24 \pm 0.21$, possibly), RSAbest (RST: $-0.11 \pm 0.22$, possibly trivial; CG: $-0.08 \pm 0.09$, very likely trivial), and RSAmean (RST: $-0.23 \pm 0.20$, possibly; CG: $-0.15 \pm$ 0.10 , likely trivial).

\begin{tabular}{|c|c|c|c|c|c|}
\hline \multicolumn{6}{|c|}{ Training program for both groups. } \\
\hline Week & $\begin{array}{l}\text { Sets } \\
(n)\end{array}$ & $\begin{array}{l}\text { Repetitions } \\
\text { (n) }\end{array}$ & $\begin{array}{l}\text { Recovery } \\
\text { between } \\
\text { repetitions } \\
\text { (s) }\end{array}$ & $\begin{array}{c}\text { Recovery } \\
\text { between } \\
\text { sets } \\
\text { (min) }\end{array}$ & Exercises \\
\hline \multicolumn{6}{|c|}{ Pre-Tests } \\
\hline 1 & 1 & 6 & 25 & - & $\begin{array}{l}\text { *8 s drills; RSG: } 3 \text { exercises with WV } \\
(10 \% \text { BM }) \text { and } 3 \text { with EC }\end{array}$ \\
\hline 2 & 2 & 5 & 25 & 2 & $\begin{array}{l}\text { *10 s drills; RSG: } 5 \text { exercises with WV } \\
\text { (10\%BM) and } 5 \text { with EC }\end{array}$ \\
\hline 3 & 2 & 5 & 25 & 2 & $\begin{array}{l}{ }^{*} 10 \text { s drills; RSG: } 5 \text { exercises with WV } \\
(12 \% B M) \text { and } 5 \text { with EC }\end{array}$ \\
\hline 4 & 2 & 6 & 30 & 3 & $\begin{array}{l}\text { *10 s drills; RSG: } 2 \times 3 \text { exercises with WV } \\
(12 \% \mathrm{BM}) \text { and } 2 \times 3 \text { with EC }\end{array}$ \\
\hline 5 & 2 & 6 & 30 & 3 & $\begin{array}{l}{ }^{*} 10-12 \text { s drills; RSG: } 2 \times 3 \text { exercises with WV } \\
(15 \% \text { BM) and } 2 \times 3 \text { with EC }\end{array}$ \\
\hline 6 & 2 & 8 & 35 & 4 & $\begin{array}{l}{ }^{*} 10-12 \text { s drills; RSG: } 2 \times 4 \text { exercises with WV } \\
(15 \% \text { BM }) \text { and } 2 \times 4 \text { with EC }\end{array}$ \\
\hline \multicolumn{6}{|c|}{ Post-Tests } \\
\hline \multicolumn{6}{|c|}{$\begin{array}{l}\text { RST: Resisted sprint training group; }{ }^{*} \text { The unresisted training group performed the same drills, } \\
\text { but with no extra resistance added; WV: Weighted vest; EC: Elastic cords; BM: Body mass }\end{array}$} \\
\hline
\end{tabular}


Table 2

Descriptive statistics and within-group changes (with 90\% confidence interval [CI]) in repeated sprint ability, change of direction and proxies of muscle power after resisted (RST) and conventional

(CG) sprint training interventions.

\begin{tabular}{|c|c|c|c|c|c|c|c|}
\hline Measures & Groups & $\begin{array}{l}\text { Pre- } \\
\text { training }\end{array}$ & $\begin{array}{l}\text { Post- } \\
\text { training }\end{array}$ & $\begin{array}{c}\% \text { Change }(90 \% \\
\text { CI) }\end{array}$ & $\begin{array}{c}\text { ES } \\
(90 \% \mathrm{CI})\end{array}$ & $\mathrm{B} / \mathrm{T} / \mathrm{H}$ & Descriptor \\
\hline \multirow{2}{*}{$\%$ DEC (\%) } & RST & $-3.7 \pm 1.3$ & $-3.1 \pm 1.1^{*}$ & $-16.92(-1.28 ; 32.56)$ & $0.43(0.03 ; 0.82)$ & $84 / 15 / 01$ & Likely \\
\hline & CG & $-3.8 \pm 1.7$ & $-3.4 \pm 1.4^{*}$ & $-11.63(-1.30 ;-21.96)$ & $0.24(0.03 ; 0.45)$ & $62 / 38 / 00$ & Possibly \\
\hline \multirow[b]{2}{*}{ RSAmean (s) } & RST & $4.48 \pm 0.24$ & $4.42 \pm 0.22^{*}$ & $-1.30(-2.43 ;-0.16)$ & $-0.23(-0.44 ;-0.03)$ & $61 / 39 / 00$ & Possibly \\
\hline & CG & $4.52 \pm 0.33$ & $4.47 \pm 0.32^{*}$ & $-1.12(-1.85 ;-0.38)$ & $-0.15(-0.24 ;-0.05)$ & $00 / 83 / 17$ & $\begin{array}{l}\text { Likely } \\
\text { Trivial }\end{array}$ \\
\hline \multirow{2}{*}{ RSAbest (s) } & RST & $4.32 \pm 0.25$ & $4.29 \pm 0.21$ & $-0.70(-2.01 ; 0.63)$ & $-0.11(-0.33 ; 0.10)$ & $24 / 75 / 01$ & $\begin{array}{c}\text { Possibly } \\
\text { Trivial }\end{array}$ \\
\hline & $\mathrm{CG}$ & $4.35 \pm 0.34$ & $4.32 \pm 0.33$ & $-0.70(-1.41 ; 0.03)$ & $-0.08(-0.17 ; 0.00)$ & 00/98/02 & $\begin{array}{l}\text { Likely } \\
\text { Trivial }\end{array}$ \\
\hline \multirow{2}{*}{$505 \mathrm{ND}$} & RST & $3.02 \pm 0.07$ & $2.97 \pm 0.06^{*}$ & $-1.61(-2.74 ;-0.47)$ & $-0.60(-1.03 ;-0.17)$ & $94 / 06 / 00$ & Likely \\
\hline & CG & $3.00 \pm 0.07$ & $2.95 \pm 0.07^{*}$ & $-1.49(-2.22 ;-0.76)$ & $-0.64(-0.95 ;-0.32)$ & $99 / 01 / 00$ & Very likely \\
\hline \multirow{2}{*}{505 DOM } & RST & $2.95 \pm 0.07$ & $2.92 \pm 0.08^{*}$ & $-1.09(-1.84 ;-0.34)$ & $-0.41(-0.69 ;-0.13)$ & $90 / 10 / 00$ & Likely \\
\hline & CG & $2.93 \pm 0.08$ & $2.93 \pm 0.08^{*}$ & $-1.19(-2.03 ;-0.36)$ & $-0.42(-0.71 ;-0.12)$ & $89 / 11 / 00$ & Likely \\
\hline \multirow{2}{*}{$\mathrm{SLJ}(\mathrm{cm})$} & RST & $233.3 \pm 17.5$ & $245.4 \pm 15.4^{*}$ & $5.27(3.25 ; 7.32)$ & $0.63(0.39 ; 0.86)$ & $100 / 00 / 00$ & $\begin{array}{l}\text { Almost } \\
\text { Certainly }\end{array}$ \\
\hline & CG & $232.0 \pm 9.9$ & $239.5 \pm 11.4^{*}$ & $3.23(2.19 ; 4.28)$ & $0.69(0.47 ; 0.91)$ & $100 / 00 / 00$ & $\begin{array}{l}\text { Almost } \\
\text { Certainly }\end{array}$ \\
\hline \multirow[b]{2}{*}{$\mathrm{CMJ}(\mathrm{cm})$} & RST & $38.8 \pm 4.3$ & $40.6 \pm 3.5^{*}$ & $4.97(2.49 ; 7.52)$ & $0.39(0.20 ; 0.58)$ & $95 / 05 / 00$ & Very Likely \\
\hline & CG & $36.3 \pm 3.5$ & $38.7 \pm 3.5^{*}$ & $6.76(4.15 ; 9.44)$ & $0.62(0.39 ; 0.86)$ & $100 / 00 / 00$ & $\begin{array}{l}\text { Almost } \\
\text { Certainly }\end{array}$ \\
\hline \multirow{2}{*}{$\begin{array}{c}20 \text { m Sprint } \\
\text { (s) }\end{array}$} & RST & $3.09 \pm 0.12$ & $3.09 \pm 0.12^{*}$ & $-1.92(-3.28 ;-0.55)$ & $-0.46(-0.78 ;-0.13)$ & $91 / 09 / 00$ & Likely \\
\hline & CG & $3.12 \pm 0.12$ & $3.05 \pm 0.11^{*}$ & $-2.26(-3.04 ;-1.48)$ & $-0.57(-0.76 ;-0.37)$ & $100 / 00 / 00$ & $\begin{array}{l}\text { Almost } \\
\text { Certainly }\end{array}$ \\
\hline \multirow{2}{*}{$\begin{array}{c}10 \mathrm{~m} \text { Sprint } \\
\text { (s) }\end{array}$} & RST & $1.80 \pm 0.07$ & $1.77 \pm 0.05^{*}$ & $-1.25(-2.05 ;-0.45)$ & $-0.32(-0.52 ;-0.11)$ & $84 / 16 / 00$ & Likely \\
\hline & $\mathrm{CG}$ & $1.82 \pm 0.04$ & $1.78 \pm 0.05^{*}$ & $-1.79(-2.70 ;-0.88)$ & $-0.77(-1.16 ;-0.38)$ & $99 / 01 / 00$ & Very Likely \\
\hline \multirow{2}{*}{$\begin{array}{c}5 \mathrm{~m} \text { Sprint } \\
\text { (s) }\end{array}$} & RST & $1.06 \pm 0.05$ & $1.02 \pm 0.05^{*}$ & $-3.15(-4.56 ;-1.71)$ & $-0.66(-0.97 ;-0.36)$ & $99 / 01 / 00$ & Very Likely \\
\hline & $\mathrm{CG}$ & $1.07 \pm 0.03$ & $1.04 \pm 0.03^{*}$ & $-2.08(-2.95 ;-1.20)$ & $-0.67(-0.95 ;-0.38)$ & $99 / 01 / 00$ & Very Likely \\
\hline
\end{tabular}

$\%$ DEC: Percentage of decrement; RSAmean: Mean time of the repeated sprint ability (RSA) test;

RSA $A_{\text {best: }}$ Best time of the RSA test; ND: Non-Dominant side; DOM: Dominant side; SLJ:

Standing long jump; CMJ: Countermovement jump; ${ }^{*}$ Based on two-way repeated measures

ANOVA, the analysis indicates that there was a significant main effect of "time" $(p<0.05)$;

B: beneficial; T: trivial; H: harmful. 


\section{Effects of RST versus CG}

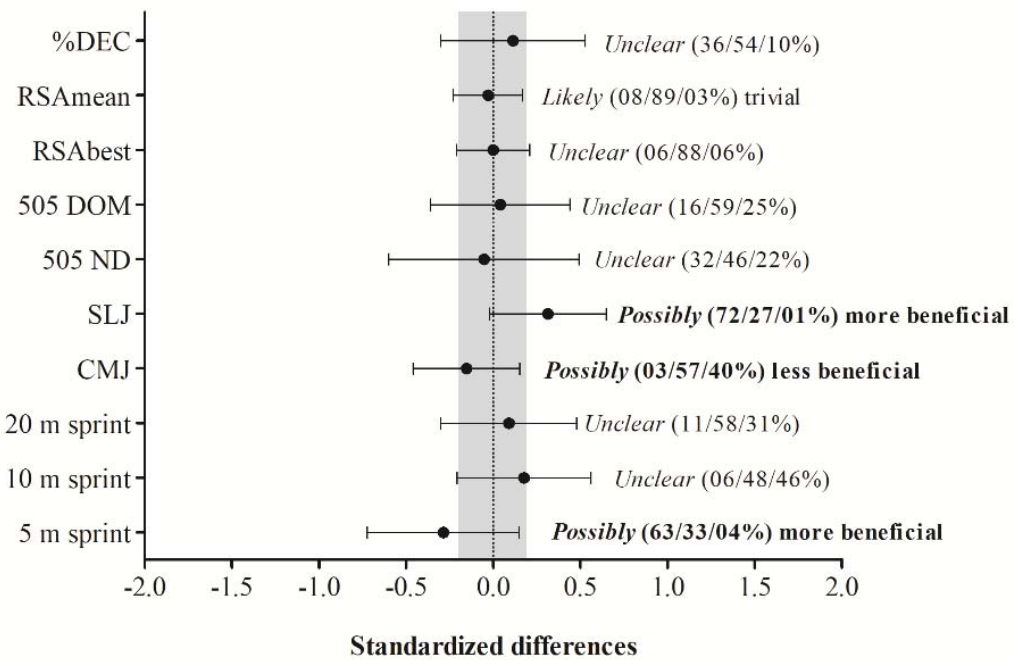

Figure 1

Effects of Resisted Sprint Training (RST) versus Conventional Sprint Training (CG) on repeated sprint ability, change of direction speed, standing long jump (SLJ), countermovement (CMJ),

and running sprint performances. Bars indicate uncertainty in the true mean changes (with 90\% confidence limits). Grey area represents the smallest worthwhile change.
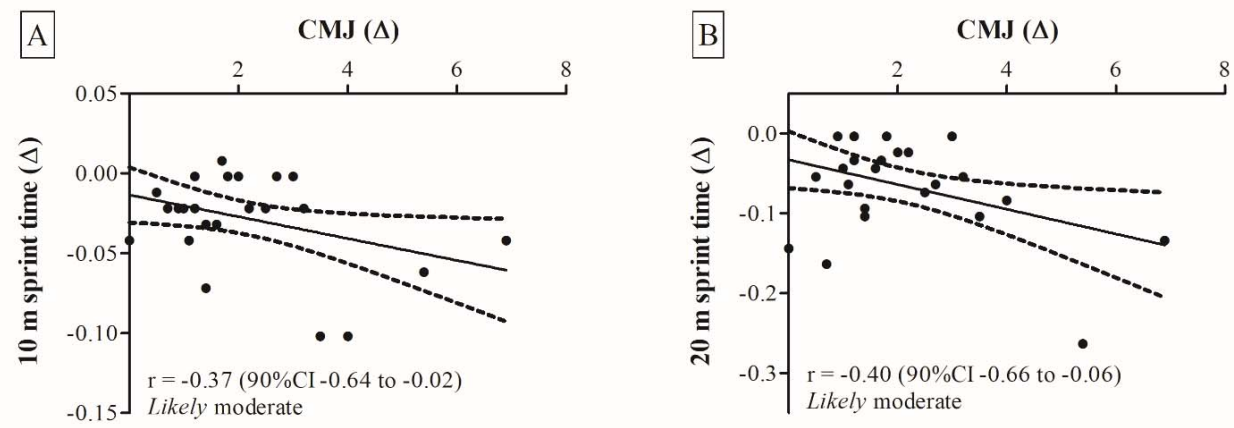

$\mathrm{C}$

$\operatorname{SLJ}(\Delta)$

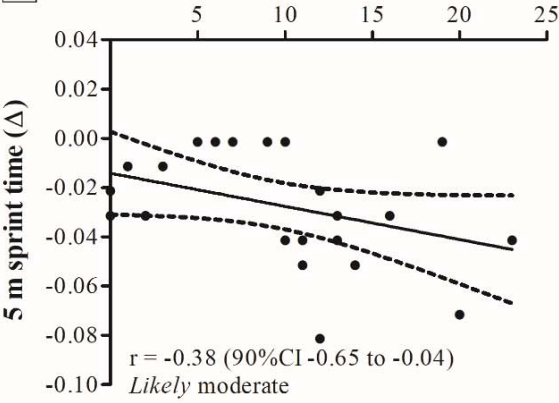

D

SLJ $(\Delta)$

Figure 2

Within-player correlations of the absolute changes $(\Delta)$ in standing long jump (SLJ) and countermovement jump (CMJ) with absolute changes $(\Delta)$ in running sprint performances. 
Figure 1 shows between-group changes over time. Compared to the CG group, improvements in the SLJ $(\mathrm{ES} \pm 90 \% \mathrm{CI}=0.31 \pm$ 0.34 ) and $5 \mathrm{~m}$ sprint time (ES $\pm 90 \% \mathrm{CI}=0.29 \pm$ 0.43 ) were possibly (chance of a greater real effect $>$ $60 \%$ ) larger in the RST group. The magnitudes of differences were rated as small. There were no substantial differences (unclear effects) for the changes in \%Dec, RSAbest, 505 ND, 505 DOM, 10 and $20 \mathrm{~m}$ sprint times between both training groups. Finally, between-group differences in the change of RSAmean were likely trivial (ES $\pm 90 \% \mathrm{CI}$ $=-0.03 \pm 0.20$ ).

Figure 2 shows the relationship between physical fitness indices. Within-player correlations between absolute changes in the SLJ and the $\mathrm{CMJ}$ with absolute changes in running sprint performances were obtained when pooling the data of RST and CG groups. There were likely moderate correlations between changes in the $\mathrm{CMJ}$ and the SLJ with some selected changes in 5 $\mathrm{m}, 10 \mathrm{~m}$ and $20 \mathrm{~m}$ sprint time.

\section{Discussion}

Findings of this study revealed that both training regimes resulted in small-to-moderate improvements in acceleration and sprint abilities $(5,10$ and $20 \mathrm{~m})$, horizontal and vertical jump performances, COD pivoting on both, the nondominant (moderate effect) and the dominant (small effect) foot, and \%Dec (small effects) during a RSA test, in male junior tennis players. Between-group comparisons showed that the SLJ and $5 \mathrm{~m}$ sprint time improved more in the RST group compared with the CG group. Overall, these findings partially confirm that RST induces larger physical fitness improvements compared to unresisted sprint training.

To the best of our knowledge, there are no studies available that have compared the effects of unresisted training with those of resisted sprint training in young tennis players. Of note, training-induced changes in this study ranged from 1.2 to $3.2 \%$ for sprint performance, irrespective of the training regime. This range is similar to findings from previous studies which examined the effects of RST using different training equipment (i.e., elastic bands, weighted sleds, vests) (Alcaraz et al., 2018; Clark et al., 2010).

The observed improvements in both experimental groups can most likely be explained with primarily neural adaptive processes (e.g., motoneuron excitability) (Ross et al., 2001) which might have caused enhanced muscle force production and movement velocity (Perrey et al., 2010). With reference to previous research, the use of weighted vests and elastic cords aims at eliciting greater vertical and horizontal net ground reaction forces during speed or agility drills, respectively (Clark et al., 2010; Rey et al., 2017). In this study, we could not find an additional effect of RST compared with CG possibly due to the low resistance that was used in RST (Petrakos et al., 2016). According to Petrakos et al. (2016), moderate loads with 10.0 to $19.9 \%$ of the individual body mass $(10-15 \%$ in the present study) seem not to be sufficient to produce extra effects compared with unresisted sprint training. Regarding the resistance offered by the elastic cord, we allowed no more than $10 \%$ velocity reductions in the designed training drills in order to preserve their biomechanical characteristics (e.g., stretch-shortening cycle participation) (Gil et al., 2018), but at the same time induce greater lower-limb power and force production (Alcaraz et al., 2018). However, due to the lack of control over the load applied in the exercises, it can be hypothesized that the tension generated through the elastic cords cannot be kept constant during the entire exercise (Gil et al., 2018). This may have resulted in a limited accuracy in overload control.

In spite of the lack of significant differences between training groups, meaningful differences between RST and CG were observed for the $5 \mathrm{~m}$ sprint change. These results are in line with previous research reporting that RST (i.e., using sled towing and weighted vests) was effective in improving kinetics and kinematics during short bouts of accelerations (Alcaraz et al., 2018; Monte et al., 2017). A reason for this difference between groups could be related to the greater increase in the capacity to produce anterior-posterior force application during RST.

Since fitness demands in tennis include multiple accelerations, decelerations and COD performance, the observed gains in the 5-0-5 test in both the RST $(1.1 \%$ and $1.6 \%$ for the dominant and non-dominant sides, respectively) and the CG group $(1.2 \%$ and $1.5 \%$ for the dominant and nondominant sides, respectively) seem to be 
practically relevant for competitive performance. We are not aware of previous studies that reported positive changes in COD after combining weighted vest and elastic cord training while performing short sprints (combined with multiple COD). Otero-Esquina et al. (2017) reported gains in linear and COD sprint when combining strength training exercises (i.e., fullback squat, leg curl on a flywheel device, plyometrics) and sled towing in soccer players. The combination of elastic cords with weighted vests impacted more on the COD pivoting on the non-dominant limb, in which a moderate effect was noticed, compared to the small effect on the dominant limb. A possible explanation for this finding is that this limb had lower initial ability to perform COD, and hence there was a better chance to improve this leg's strength, power and stiffness with adequate training stimuli.

Besides improving sprint ability, both unresisted and resisted sprint training resulted in increased SLJ (RST: $+5.3 \%$; CG: $+3.2 \%$ ) and CMJ (RST: $+5 \%$; CG: $+6.8 \%$ ) performances. This is in agreement with the notion that improved sprint performance is to a high degree related to enhancements in lower-limb muscle power production in both vertical and horizontal directions (Loturco et al., 2018), inferred from jump height and distance, respectively. In fact, the observed significant correlations between increases in SLJ and CMJ performance and reduced sprint times from pre- to post-training confirm the neuro-mechanical relationships between these qualities. Moreover, since there is a transfer of increment in the SLJ to acceleration performance over short distances (e.g., $5 \mathrm{~m}$ ) (Loturco et al., 2015), this may explain why the SLJ and acceleration over $5 \mathrm{~m}$ improved more in the RST group compared with the CG group. It is apparent that force and power production in the anterior-posterior axis were optimized by the use of additional (but light) resistance during training drills performed by tennis players. However, unresisted sprint training was also effective in enhancing vertical and horizontal jump performances, and this partly confirms the results from other studies conducted with individual and team sports athletes (Fernandez-Fernandez et al., 2015; Gil et al., 2018; Spinks et al., 2007).

Since training of competitive tennis players should focus on improving their ability to repeatedly perform high-intensity efforts and to recover rapidly between bouts (FernandezFernandez et al., 2012), training strategies that aim to improve qualities such as RSA could be significant for tennis. The results of our MBIbased approach revealed that only RST improved RSAmean (-1.3\%; small effect size), although between-group delta changes were not different. Previously, it was shown that futsal players displayed greater RSA improvements in a group combining resistance training with loaded change of direction drills, compared to a group that solely conducted resistance training and a control group (Torres-Torrelo et al., 2018). Torres-Torrello et al. (2018) observed that improvements in RSAmean were accompanied with shorter ground contact time during sprints, suggesting an increase in the rate of force development. Unfortunately, we did not conduct kinematic sprint analysis to elucidate and confirm this finding. Both RST and CG resulted in better \%Dec during the RSA test, without any changes in RSAbest. However, the response of this index to training should be viewed with caution (Bishop et al., 2011), since, for example, a detraining period can impair RSAbest and artificially improve \%Dec. Of note, in this study, tennis players of both groups showed similar changes in \%Dec, in the expected direction, given that they followed the respective training regimes. However, due to the positive adaptation in RSAmean that was observed in RST only with the MBI approach, it is advisable that strength and conditioning professionals adopt resisted short accelerations and sprints in their routines to improve this performance relevant physical quality for tennis (Fernandez-Fernandez et al., 2012).

In summary, both conventional (unresisted) and resisted sprint and COD training drills implemented for 6 weeks in junior tennis players appeared to be effective in improving key physical fitness components for youth tennis, such as acceleration speed, horizontal and vertical jump ability, change of direction and repeated sprint ability. However, there were small, but meaningful advantages of performing resisted drills to improve horizontal jump and 5-m acceleration, compared to unresisted sprint training drills.

It is also important to highlight possible limitations of this study. The interpretation and 
application of our data have to be done with caution as our findings are specific to a population of young male tennis players. Regarding the applied training methods, it should be acknowledged that the use of elastic cords does not allow to adequately follow the overload principle because the load cannot be kept constant. Furthermore, and also related to the training equipment used here, there is evidence from recent studies to suggest that heavier loads (i.e., inducing speed reduction > 10-15\% established here) are needed to induce performance improvements, in particular if the goal is to improve sprint performance over short distances (Kawamori et al., 2014; Morin et al., 2017; Petrakos et al., 2016).

\section{Conclusions and Practical Implications}

Based on the present results, 6 weeks of RST or unresisted sprint training appear to represent a time-efficient stimulus for physical fitness improvements in young male tennis players. Given the relatively low training volume and the low cost of training equipment, this intervention seems to be practically relevant for tennis coaches and athletes. RST and/or unresisted sprint training can easily be integrated two times per week as part of the regular inseason training. Of note, it should always be conducted prior to a tennis session (FernandezFernandez et al., 2018). Since small differences can be very important when working with elite athletes, the small advantages of RST over unresisted sprint training to improve horizontal jump and 5-m acceleration may suggest that RST should be preferred over unresisted sprint training if the goal is to improve sport-specific performance determinants in youth tennis.

\section{References}

Alcaraz PE, Carlos-Vivas J, Oponjuru BO, Martínez-Rodríguez A. The Effectiveness of Resisted Sled Training (RST) for Sprint Performance: A Systematic Review and Meta-analysis. Sports Med, 2018; 48(9): 21432165

Alcaraz PE, Elvira JLL, Palao JM. Kinematic, strength, and stiffness adaptations after a short-term sled towing training in athletes. Scand J Med Sci Sports, 2014; 24(2): 279-290

Aloui G, Hammami M, Fathloun M, Hermassi S, Gaamouri N, Shephard RJ, Chelly MS. Effects of an 8-Week In-Season Elastic Band Training Program on Explosive Muscle Performance, Change of Direction, and Repeated Changes of Direction in the Lower Limbs of Junior Male Handball Players. J Strength Cond Res, 2019; 33(7): 1804-1815

Bishop D, Girard O, Mendez-Villanueva A. Repeated-sprint ability - part II: recommendations for training. Sports Med, 2011; 41(9): 741-756

Carlos-Vivas J, Marin-Cascales E, Freitas TT, Perez-Gomez J, Alcaraz PE. Force-velocity-power profiling during weighted vest sprinting in soccer. Int J Sports Physiol Perform, 2019; 14(6): 747-756

Castro-Piñero J, Ortega FB, Artero EG, Girela-Rejón MJ, Mora J, Sjöström M, Ruiz JR. Assessing muscular strength in youth: usefulness of standing long jump as a general index of muscular fitness. J Strength Cond Res, 2010; 24(7): 1810-1817

Clark KP, Stearne DJ, Walts CT, and Miller AD. The longitudinal effects of resisted sprint training using weighted sleds vs. weighted vests. J Strength Cond Res, 2010; 24(12): 3287-3295

Cormie P, McGuigan MR, Newton RU. Developing maximal neuromuscular power: Part 1--biological basis of maximal power production. Sports Med, 2011; 41(1): 17-38

Cottle CA, Carlson LA, Lawrence MA. Effects of sled towing on sprint starts. J Strength Cond Res, 2014; 28(5): $1241-1245$

Cronin JB, Hansen KT. Strength and Power Predictors of Sports Speed. J Strength Cond Res, 2005; 19(2): 349357

Fernandez-Fernandez J, Granacher U, Sanz-Rivas D, Sarabia Marín JM, Hernandez-Davo JL, Moya M. Sequencing Effects of Neuromuscular Training on Physical Fitness in Youth Elite Tennis Players. J Strength Cond Res, 2018; 32(3): 849-856 
Fernandez-Fernandez J, Sanz-Rivas D, Kovacs MS, Moya M. In-season effect of a combined repeated sprint and explosive strength training program on elite junior tennis players. J Strength Cond Res, 2015; 29(2): 351-357

Fernandez-Fernandez J, Sanz-Rivas D, Mendez-Villanueva A. A Review of the Activity Profile and Physiological Demands of Tennis Match Play. Strength Cond J, 2009; 31(4): 15-26

Fernandez-Fernandez J, Ulbricht A, Ferrauti A. Fitness testing of tennis players: How valuable is it? $\mathrm{Br} \mathrm{J}$ Sports Med, 2014; 48(Suppl 1): i22-i31

Fernandez-Fernandez J, Zimek R, Wiewelhove T, Ferrauti A. High-intensity interval training vs. repeatedsprint training in tennis. J Strength Cond Res, 2012; 26(1): 53-62

Gallo-Salazar C, Del Coso J, Barbado D, Lopez-Valenciano A, Santos-Rosa FJ, Sanz-Rivas D, Moya M, Fernandez-Fernandez J. Impact of a competition with two consecutive matches in a day on physical performance in young tennis players. Appl Physiol Nutr Metab, 2017; 42(7): 750-756

Gil S, Barroso R, Crivoi do Carmo E, Loturco I, Kobal R, Tricoli V, Ugrinowitsch C, Roschel H. Effects of resisted sprint training on sprinting ability and change of direction speed in professional soccer players. J Sports Sci, 2018; 36(17): 1923-1929

Girard O, Mendez-Villanueva A, Bishop D. Repeated-sprint ability - part I: factors contributing to fatigue. Sports Med, 2011; 41(8): 673-694

Girard O, Micallef JP, Millet GP. Lower-limb activity during the power serve in tennis: effects of performance level. Med Sci Sports Exerc, 2005; 37(6): 1021-1029

Hopkins WG, Marshall SW, Batterham AM, Hanin J. Progressive statistics for studies in sports medicine and exercise science. Med Sci Sports Exerc, 2009; 41(1): 3-13

Hopkins WG. A spreadsheet for deriving a confidence interval, mechanistic inference and clinical inference from a P value. Sportscience, 2007; 11: 16-21

Hrysomallis C. The effectiveness of resisted movement training on sprinting and jumping performance. $J$ Strength Cond Res, 2012; 26(1): 299-306

Kawamori N, Newton R, Nosaka K. Effects of weighted sled towing on ground reaction force during the acceleration phase of sprint running. J Sports Sci, 2014; 32(12): 1139-1145

Kovacs M. Applied physiology of tennis performance. Br J Sports Med, 2006; 40(5): 381-385

Kovacs M. Tennis Physiology: Training the competitive athlete. Sports Med, 2007; 37(3): 189-198

Loturco I, Kobal R, Kitamura K, Cal Abad CC, Faust B, Almeida L, Pereira LA. Mixed Training Methods: Effects of Combining Resisted Sprints or Plyometrics with Optimum Power Loads on Sprint and Agility Performance in Professional Soccer Players. Front Physiol, 2017; 8: 1034

Loturco I, Pereira LA, Kobal R, Nakamura, FY. Using Loaded and Unloaded Jumps to Increase Speed and Power Performance in Elite Young and Senior Soccer Players. Strength Cond J, 2018; 40(3): 95-103

Loturco I, Pereira L, Kobal R, Zanetti V, Kitamura K, Abad CCC, Nakamura F. Transference effect of vertical and horizontal plyometrics on sprint performance of high-level U-20 soccer players. J Sports Sci, 2015; 33(20): 2182-2191

Markovic G, Dizdar D, Jukic I, Cardinale M. Reliability and Factorial Validity of Squat and Countermovement Jump Tests. J Strength Cond Res, 2004; 18(3): 551-554

Martínez-Valencia MA, Romero-Arenas S, Elvira JLL, González-Ravé JM, Navarro-Valdivielso F, Alcaraz PE. Effects of Sled Towing on Peak Force, the Rate of Force Development and Sprint Performance During the Acceleration Phase. J Hum Kinet, 2015; 46(1): 139-148

Monte A, Nardello F, Zamparo P. Sled Towing: The Optimal Overload for Peak Power Production. Int J Sports Physiol Perform, 2017; 12(8): 1052-1058

Morin JB, Petrakos G, Jiménez-Reyes P, Brown SR, Samozino P, Cross MR. Very-Heavy Sled Training for Improving Horizontal-Force Output in Soccer Players. Int J Sports Physiol Perform, 2017; 12(6): 840-844 
Otero-Esquina C, de Hoyo Lora M, Gonzalo-Skok Ó, Domínguez-Cobo S, Sánchez H. Is strength-training frequency a key factor to develop performance adaptations in young elite soccer players? Eur J Sport Sci, 2017; 17(10): 1241-1251

Perrey S, Racinais S, Saimouaa K, Girard O. Neural and muscular adjustments following repeated running sprints. Eur J Appl Physiol, 2010; 109(6): 1027-1036

Petrakos G, Morin JB, Egan B. Resisted Sled Sprint Training to Improve Sprint Performance: A Systematic Review. Sports Med, 2016; 46(3): 381-400

Rey E, Padrón-Cabo A, Fernández-Penedo D. Effects of Sprint Training With and Without Weighted Vest on Speed and Repeated Sprint Ability in Male Soccer Players. J Strength Cond Res, 2017; 31(10): 2659-2666

Ross A, Leveritt M, Riek S. Neural influences on sprint running: training adaptations and acute responses. Sports Med, 2001; 31(6): 409-425

Spencer M, Bishop D, Dawson B, Goodman C. Physiological and metabolic responses of repeated-sprint activities: specific to field-based team sports. Sports Med, 2005; 35(12): 1025-1044

Spinks CD, Murphy AJ, Spinks WL, Lockie, RG. The effects of resisted sprint training on acceleration performance and kinematics in soccer, rugby union, and Australian football players. J Strength Cond Res, 2007; 21(1): 77-85

Torres-Torrelo J, Rodríguez-Rosell D, Mora-Custodio R, Pareja-Blanco F, Yañez-García JM, González-Badillo JJ. Effects of Resistance Training and Combined Training Program on Repeated Sprint Ability in Futsal Players. Int J Sports Med, 2018; 39(7): 517-526

\section{Corresponding author:}

\section{Jaime Fernandez-Fernandez, Phd}

Department of Physical Activity and Sports Sciences.

Universidad de León.

Campus de Vegazana s/n, 24071 - Spain; Phone: (0034)987293026

E-mail: jaime.fernandez@unileon.es 